\title{
Predictability of stock markets with disequilibrium trading
}

\author{
WOJCIECH W. CHAREMZA*, KALVINDER SHIELDS** and \\ ANNA ZALEWSKA*** \\ * University of Leicester, UK \\ ** University of Melbourne, Australia \\ *** Maastricht University, The Netherlands
}

This paper analyses the predictability of a hypothetical market with freely negotiated prices on which exists a censoring of one-period returns which are in excess of an arbitrary level ('floor' and 'ceiling'). It is shown that the expected value of returns (adjusted for drift) conditional on last period information regarding the censoring are equal to zero (and therefore the market is not predictable in mean) if there is no intertemporal spillover on the market. A simple simulation model is proposed and applied for the analysis of the effects of intertemporal and cross-spillovers resulting from quantity constraints. Statistical predictability tests are proposed, based on the corrected Student- $t$ statistic of a regression of returns of some information concerning the previous censoring. An illustrative empirical analysis of six main time series of returns on the Warsaw Stock Exchange confirms their ex-ante, but not ex-post, predictability.

Keywords: efficient markets, East European financial markets, censored returns

JEL Classifications: $\mathrm{C} 22, \mathrm{C} 24, \mathrm{G} 12$

\section{INTRODUCTION}

One of the primary issues of the time series analysis of financial markets is the predictability of stock returns. It is generally claimed that if returns are not predictable (in mean), the market is, is some sense, efficient. The literature on this subject is voluminous (for reviews of literature on various types of research into the efficient market hypothesis see, e.g. Baillie (1989), LeRoy (1989) and, for the non-linear generalisation of this concept, Peters (1991)). Results of empirical testing of predictability of returns on various stock markets are often inconclusive; however, it is often admitted that proving the existence, or non-existence of such predictability, is not feasible (see e.g. the seminal papers by Fama (1965) followed by Fama (1970) and (1991), Grossman and Stiglitz (1980), Granger (1992), and Williams (1994) for a historical review). Nevertheless, in the context of emerging financial markets, the results more strongly favour the hypothesis of non-predictability (see e.g. Claessens et al. (1995), Richards (1996)).

Corresponding author: Wojciech W. Charemza, University of Leicester, Department of Economics, University Road, Leicester LE2 4GJ, UK. 
The fact whether a market is predictable or not does not decide upon the existence, or non-existence, of speculation. Stock market speculation is generally regarded as a negative phenomenon which should be eliminated or at least reduced. One of the well-known attempts to curb speculation is by introducing so-called 'par casier' or 'Einheitskurs' system of quotations, that is where upper and lower limits on price movements are introduced. In one of its variations, if the price calculated at the end of a trading session, reaches its lower or upper limit, it is not allowed to move further and trading is either suspended, or the transactions volume is reduced proportionally on the 'long' side. Such a system was implemented on the Warsaw Stock Exchange in 1991 and similar mechanisms of price control have been also used on other stock exchanges, both on the emerging (Shanghai, Vilnius, Riga) and mature (Paris) markets. In this paper, we call this a disequilibrium trading system.

We show that the fact that the upper and lower price limits are imposed might not necessarily be a source for market predictability. In particular, if there is some kind of funds withdrawal process due to a quantity spillover in relation to other stocks or to non-market assets, the market might not be predictable. We have examined three simple regression tests and suggested a non-parametric correction which we find, in some circumstances, improves their power. We also show preliminary results of testing for market predictability on the Warsaw Stock Exchange.

\section{TRADERS' BEHAVIOUR UNDER THE EXPECTED QUANTITY CONSTRAINT}

In order to explain traders' behaviour on a stock market where a binding constraint on a quantity of shares traded is expected, let us assume that a representative trader faces in time 0 the intertemporal allocation decision of how much to invest in asset $\boldsymbol{x}$ in the present time 0 , and how much to invest in future time 1 . Let us denote these quantities as $\boldsymbol{x}_{0}$ and $\boldsymbol{x}_{1}$ respectively. For this security the 'Einheitskurs' system is implemented. Note that according to this price-setting process, since the price is announced at the end of the trading day, the trader does not have the opportunity of withdrawing, or indeed altering, his demand (supply) at time $t$. The representative trader's utility function, simplified substantially for the sake of clarity of exposition, is given as:

$$
U=U\left(x_{0}, x_{1}, \Omega\right)
$$

with the financial constraint:

$$
\bar{\Omega}=p_{0} x_{0}+p_{1} x_{1},
$$

where $p_{0}$ is the observed price for $\boldsymbol{x}$ in time $0, p_{1}$ is the price for $x$ expected in time 0 for time 1 , and $\bar{\Omega}$ stands for the initial endowment of wealth $\Omega$ of the trader. Maximisation of (1) with the financial constraint gives the notional (Walrasian) demand functions for expressing the amount of $\boldsymbol{x}$ the trader is willing to trade 
in periods 0 and 1 in the absence of any constraints on the market (see e.g. Benassy (1982)):

$$
\begin{aligned}
& \hat{x}_{0}^{d}=\hat{x}_{0}^{d}\left(\bar{\Omega}, p_{0}, p_{1}\right), \\
& \hat{x}_{1}^{d}=\hat{x}_{1}^{d}\left(\bar{\Omega}, p_{0}, p_{1}\right) .
\end{aligned}
$$

The Walrasian quantities satisfy the financial constraint, and therefore the prices and quantities for time 1 can be represented as being conditional on information related to time 0 as:

$$
p_{1} x_{1}=\bar{\Omega}-p_{0} \hat{x}_{0}^{d} .
$$

Given that the right-hand side of (3) depends solely on prices and the initial financial endowment, then, under the standard assumptions of the efficient markets (see e.g. LeRoy (1989)), the expected price $p_{1}$ can be given by the martingale hypothesis:

$$
p_{1}=p_{0} .
$$

Suppose now that, in time 0 , there is a binding transactional constraint $\bar{x}_{0}$ appearing in the market as a result of the 'Einheitskurs' system, that is:

$$
x_{0}=\bar{x}_{0} .
$$

Let us assume that this constraint is not manipulable. Then, maximising (1) under the financial constraint (2) and the quantity constraint (5) gives the following effective demand function for asset $x$ in time 1, which is also a function of the quantity constrained asset in time 0 , i.e.:

$$
x_{1}^{d}=x_{1}^{d}\left(\bar{\Omega}, p_{0}, p_{1}, \bar{x}_{0}\right) .
$$

It follows that the first two terms of the expansion of $x_{1}^{d}$ in a Taylor series around the Walrasian notional gives:

$$
x_{1}^{d} \approx x_{1}^{d}\left(\bar{\Omega}, p_{0}, p_{1}, \hat{x}_{0}^{d}\right)+\left(\bar{x}_{0}-\hat{x}_{0}^{d}\right) \cdot C_{x}=\hat{x}_{1}^{d}+\left(\bar{x}_{0}-\hat{x}_{0}^{d}\right) \cdot C_{x},
$$

where:

$$
C_{x}=\frac{\partial x_{1}^{d}}{\partial \bar{x}_{0}}\left(\bar{\Omega}, p_{0}, p_{1}, \hat{x}_{0}^{d}\right) .
$$

The function $C_{x}$ measures the intertemporal spillover which appears on the market due to the presence of excess demand in period 0 (see e.g. Quandt (1988)). The expected sign of $C_{X}$ is nonpositive since the trader is likely to increase trading in security $\boldsymbol{x}$ in time 1 by a portion of the demand which was not satisfied in time 0 . Hence, if $C_{x}$ tends towards zero, then this means that the trader decides to withdraw a relatively large part of excess demand (where excess demand represents the part of demand not satisfied and equals $\left(\bar{x}_{0}-\hat{x}_{0}^{d}\right)$ ) from the market for $\boldsymbol{x}$ into 
wealth. Let us call such a withdrawal a cross-spillover effect. We can consider in more detail the cases for the polar values of $C_{x}$ and the implications for the martingale hypothesis (4) assuming that the function $C_{X}$ is linearly homogenous in price, i.e. $C_{x}^{*}=p_{0} C_{x}$.

In the case of a full cross-spillover effect and no intertemporal spillover, where any excess demand is completely withdrawn from the market for security $\boldsymbol{x}$, then this is the case for which $C_{x}^{*}=0$. This results in the following:

$$
x_{1}^{d} \approx \hat{x}_{1}^{d},
$$

with the financial constraint being given by:

$$
p_{1} x_{1}=\bar{\Omega}-p_{0} \hat{x}_{0}^{d},
$$

which is the same as the constraint in expression (3). Note that this financial constraint does not contain any information regarding the constraint $\bar{x}_{0}$. This implies that despite the presence of a constraint on the market, price expectations are formed solely on the basis of the prices observed in time 0 , and the martingale hypothesis (4) can be maintained.

The other extreme case of $C_{x}^{*}=-1$, reflects the situation of a full intertemporal spillover and no cross-spillover effect and gives:

$$
x_{1}^{d} \approx \hat{x}_{1}^{d}-p_{0} \bar{x}_{0}+p_{0} \hat{x}_{0}^{d},
$$

with the corresponding financial constraint:

$$
p_{1} x_{1}=\bar{\Omega}-p_{0} \bar{x}_{0} .
$$

Since $\bar{x}_{0}$ does not depend on the price, then, in general, the martingale hypothesis cannot be maintained. It is, however, possible to find a 'virtual' price $\bar{p}_{0}$ which would support both the financial constraint and the Walrasian demand, such that:

$$
p_{0} \bar{x}_{0}=\bar{p}_{0} \hat{x}_{0}^{d} .
$$

If such a price was known, then:

$$
p_{1} x_{1}=\bar{\Omega}-\bar{p}_{0} \hat{x}_{0}^{d},
$$

indicates that the expected price can be given by:

$$
p_{1}=\bar{p}_{0} .
$$

\section{A MODEL OF A DISEQUILIBRIUM TRADING SYSTEM}

The direct consequence of the analysis of traders' behaviour presented in section 2 of this paper is a model of price setting which reflects both the fact that prices are not allow to move beyond a pre-set boundary, and also that a price in time $t$, may, even if not constrained, be affected by a previous hit of a price into the boundary. It is initially assumed that the process representing the observed prices, $p_{t}$, 
and the underlying (unrestricted) prices, $p_{t}^{*}$, in their logarithms on a market with disequilibrium trading, is given as:

$$
\begin{aligned}
& p_{t}^{*}=\theta p_{t-1}^{*}+(1-\theta) p_{t-1}+\epsilon_{t} ; \quad \theta \in[0,1] \\
& p_{t}=\left\{\begin{array}{cll}
p_{t}^{*} & \text { if } & \left|p_{t}^{*}-p_{t-1}\right|<\omega \\
\omega+p_{t-1} & \text { if } & p_{t}^{*}-p_{t-1}>\omega \\
-\omega+p_{t-1} & \text { if } & p_{t}^{*}-p_{t-1}<-\omega
\end{array}\right.
\end{aligned}
$$

where $\varepsilon_{t}$ is a martingale difference sequence and constant $\omega$ represents the upper and lower limit for the allowable price movement.

Ignoring the possible payments of dividends, returns are defined as $r_{t}=p_{t}-p_{t-1}$. From (6) and (7) it is clear that:

$$
r_{t}=\operatorname{sgn}\left(\varepsilon_{t}\right) \cdot \min \left(\omega,\left|\varepsilon_{t}\right|\right),
$$

or, in another words the returns are equal to $\varepsilon_{t}$, whenever the constraint is not binding, or to the upper or lower boundary for price movement if the constraint is binding. The artificial process above seems to represent the empirical series of returns from a market with disequilibrium trading satisfactorily; Fig. 1 compares time series of returns simulated by the process (6)-(7) with $\theta=0.5$ and $\varepsilon_{t} \sim$ $\operatorname{IIDN}(0,1)$, and returns of an exemplary security from the Warsaw Stock Exchange (returns on Próchnik shares) for 443 returns from a session to session in the period from the creation of Warsaw Stock Exchange (12 Apri1 1991) until the end of 1993. A detailed description of the empirical data is found in Section 5 . The similarity of the series is evident. The outliers exceeding the $10 \%$ limit for the Próchnik returns result from occasional suspension of the limit.

The weight $\theta$ which aggregates the lagged underlying and observed processes plays an important role in the evaluation of market predictability. If neither floor nor ceiling is hit, then the underlying process becomes equal to the observed process for any $\theta$ and returns are just martingale differences.

If one of the boundaries is hit, then $p_{t} \neq p_{t}^{*}$ and $\theta$ decides about the nature of the market. In the extreme case, where $\theta=1$, there is a recursive influence of the underlying process on that of the observed prices, without a feedback from the constrained price. In practice it means that, despite information that a boundary was hit, no capital is to be reallocated to or from the market. Recalling the analysis given in Section 2, $\theta=1$ corresponds to the case of the full intertemporal and spillover and no cross-spillover. Clearly, the returns $p_{t}-p_{t-1}^{*}$ are not martingale differences if $p_{t} \neq p_{t}^{*}$.

If $\theta=0$, then there is a full feedback from the constrained price to the underlying price and there is a fully binding quantity constraint. Effective demand becomes equal to the notional demand, i.e. that of the Drèze sense (see Drèze (1975), Quandt (1988, pp. 165-166)), so that demand carried forward from time $t-1$ to time $t$ is zero even if there is (positive or negative) excess demand on the market in time $t-1$. This represents the case discussed in the previous section where there is a full cross-spillover and no intertemporal spillover. For the intermediate case $0<\theta<1$, there is a partial withdrawal of funds from the market under disequilibrium trading, either on the supply or demand side. 
Observed Series

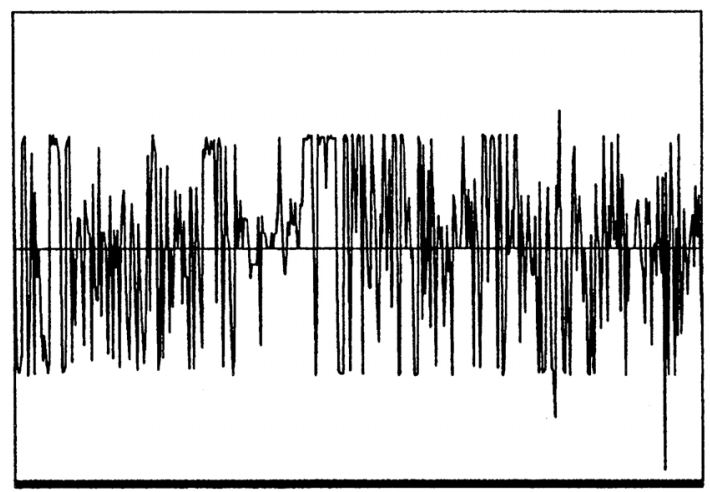

\section{Simulated Series}

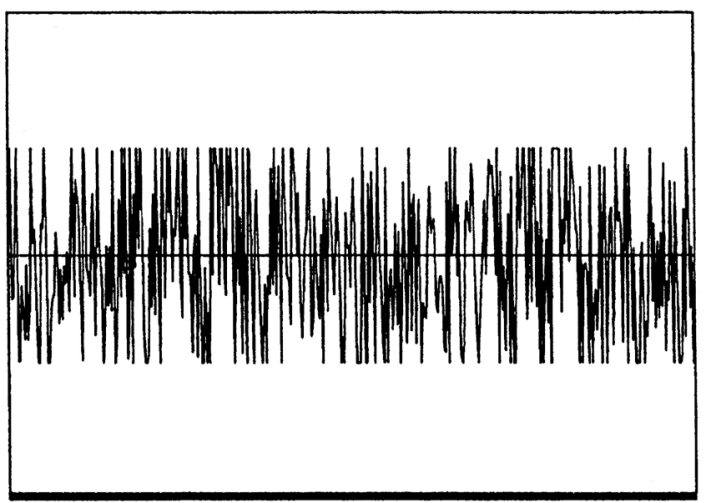

Fig. 1. Returns from a typical at the Warsaw Stock Exchange (Próchnik) and the simulated disequilibrium trading process.

It is shown in the Appendix that the market described by (6)-(7) is predictable in mean if $0<\theta \leq 1$, and hence inefficient, in the sense that the expected value of returns conditional on information that the upper (lower) bound was hit, is non-zero. If $\theta=0$, the market is not predictable. The strongest possible case of predictability in model (6)-(7) is where $\theta=1$. The situation where $0<\theta<1$ represents an intermediate situation, where $\theta$ can be interpreted as a measure of market predictability.

\section{THREE SIMPLE PREDICTABILITY TESTS}

For an empirical investigation of a market with disequilibrium trading, it seems to be important to decide whether the parameter $\theta$ in (6) is equal to zero. Recall that, 
if $\theta=0$, then the market is not predictable in mean. The first simple test can be based on a linear regression approximation to $E\left(r_{t} \mid \varepsilon_{t-1}>\omega\right)$, that is a regression of (de-meaned) returns on dummy variables representing a hit of the barrier in time $t-1$ (Test I), i.e.:

$$
r_{t}=\beta_{1} d_{t-1}^{+}+\beta_{2} d_{t-1}^{-}+\text {error }
$$

where:

$$
d_{t}^{+}=\left\{\begin{array}{lll}
1 & \text { if } & \varepsilon_{t}>\omega \\
0 & & \text { otherwise }
\end{array}, \quad d_{t}^{-}=\left\{\begin{array}{lll}
1 & \text { if } & \varepsilon_{t}<-\omega \\
0 & & \text { otherwise }
\end{array} .\right.\right.
$$

Regression (8) can be estimated by ordinary least squares $(O L S)$ and then the hypotheses that $\beta_{1}=0$ and $\beta_{2}=0$ can be tested by the Student- $t$ test.

Another possibility is to use a simplification of this test by exploiting the symmetry of the positive and negative constraints and assuming that $\beta_{1}=\beta_{2}$. In this case regression (8) becomes (Test II):

$$
r_{t}=\beta d_{t-1}^{ \pm}+\text {error }
$$

where:

$$
d_{t}^{ \pm}=\left\{\begin{array}{lll}
1 & \text { if } & \epsilon_{1}>\omega \\
-1 & \text { if } & \epsilon_{1}<-\omega \\
0 & & \text { otherwise. }
\end{array}\right.
$$

Ideally, if any information concerning the volume of transactions not realised on the market is available, it is possible to carry out a third test where the quantitative information concerning excess demand enters the regression equation in place of the qualitative dummy variables (Test III), i.e.:

$$
r_{t}=\gamma\left(p_{t-1}^{*}-p_{t-1}\right)+\text { error } .
$$

It should be remembered that in (10) the variable $p_{t-1}^{*}-p_{t-1}$ takes a non-zero value only if a barrier was hit in time $t-1$.

The above tests are in fact ex-ante predictability tests, since they do not take into account the fact that, while a disequilibrium state occurs in time $t-1$, it might also occur in time $t$, preventing the expected abnormal profits to materialise. There is a possibility for a development of analogous ex-post predictability tests, which would concentrate on predicting abnormal returns from time $t-1$ for time $t$ if, in time $t, r_{t}=\varepsilon_{t}$. This problem is, however, beyond the scope of this paper.

Due to the censoring nature of the arguments in the above tests, it would be interesting to evaluate the empirical size and power of these tests. In particular, because of the difference in dispersion of the arguments and the dependent variable for a high frequency of censoring, the power of these tests may be affected for large values of $\theta$. In order to assess this, we have performed a series of naive Monte Carlo experiments, consisting of the computation of empirical frequencies of rejection of the null hypothesis that $\beta_{1}=0$ (for Test I), $\beta=0$ (for Test II) and $\gamma=0$ (for Test III). The data generating process for these experiments is given 
by equations (6)-(7), with $\varepsilon_{t} \sim \operatorname{IIDN}(0,1), \omega=\ln (1.1)$, which corresponds to the $10 \%$ upper and lower limit on price movements, usually imposed at the Warsaw Stock Exchange, with sample sizes equal to 100, 250 and 500 and $\theta$ changing from 0 to 1.0 by 0.1 increments. Hence, the total number of simulation experiments is 33 and, in each, 5000 replications were performed.

The empirical frequency of rejections of the null hypothesis of a standard Student- $t$ at the $5 \%$ level of significance is shown in Fig. 2. The Figure indicates that size of the Student- $t$ test is not affected, that is the empirical frequency of rejections for $\theta=0$ (that is, for the case where the market is not predictable) is close to $5 \%$ for all three tests. Generally, the most powerful is Test III; this is not surprising, since it uses quantitative rather than qualitative information concerning excess demand. The empirical power of all the tests increases until $\theta$ reaches high values, where it then starts to decrease. This can be explained by considering a simple regression Student- $t$ statistic from the regression equation:

$$
y_{t}=\alpha^{\prime} x_{t}+u_{t},
$$

where $\alpha$ is the vector of the regression coefficients $x_{t}$ are the explanatory variables and $u_{t}$, is the error term. All the three tests discussed in this paper can be represented by this regression. For the $i$-th regression coefficient, the Student- $t$ statistic is given by:

$$
t_{i}=\frac{\hat{\alpha}_{i}}{S_{\alpha i}}
$$

where $\hat{\alpha}_{i}$ is the $O L S$ estimate of the $i$-th element in $\alpha, S_{\alpha i}=S_{u} \cdot \sqrt{\operatorname{diag}_{i i}\left(X^{\prime} X\right)^{-1}} ; S_{u}$ is the standard error of the residuals, and $X$ is the matrix of observations of the variables in $x_{t}$. The most probable reason for the decrease of power for large $\theta$, is that as the frequency of the barrier hits is increasing, it reduces the variation of the observed returns. At the same time, the variation of the dependent variables is either decreasing at a slower pace (for Tests I and II), or is increasing (for Test III), forcing the estimated regression coefficient $\hat{\alpha}_{i}$ to become smaller. This simultaneously causes the corresponding elements of $\operatorname{diag}_{i i}\left(X^{\prime} X\right)^{-1}$ to decrease more slowly, or to increase. As a result, the Student- $t$ ratio, given by the expression above, tends to decrease, thus affecting the power of the test.

With this in mind, it would be possible to increase the power of the Student- $t$ tests by imposing a non-parametric correction to the standard error of the estimates used for computing the $t$-ratio. This correction reflects the fact that, for a given sample size and the censoring point, the frequency of 'barrier hits' tends to increase with an increase in $\theta$. It can be given as,

$$
S_{\alpha_{i}}^{*}=S_{u} \cdot \sqrt{\operatorname{diag}_{i i}\left(X^{\prime} X\right)^{-1}} \cdot \sqrt{1-\left(\frac{N^{h}}{N}-v\right)},
$$

where $N^{h}$ is the number of barrier hits in a sample and $v=\operatorname{Pr}\left(\omega>\left|\varepsilon_{t}\right| \mid \theta=0\right.$ for the two-sided tests, that is for Test II and Test III, and $v=\operatorname{Pr}\left(\omega>\varepsilon_{t} \mid \theta=0\right)=\operatorname{Pr}(-\omega<$ $\varepsilon_{t} \mid \theta=0$ ) for Test I. It can be verified that, for $\theta=0$, the expected frequency of hits is equal to $v$, hence resulting in the traditional $O L S$ Student- $t$ ratio. Therefore, 

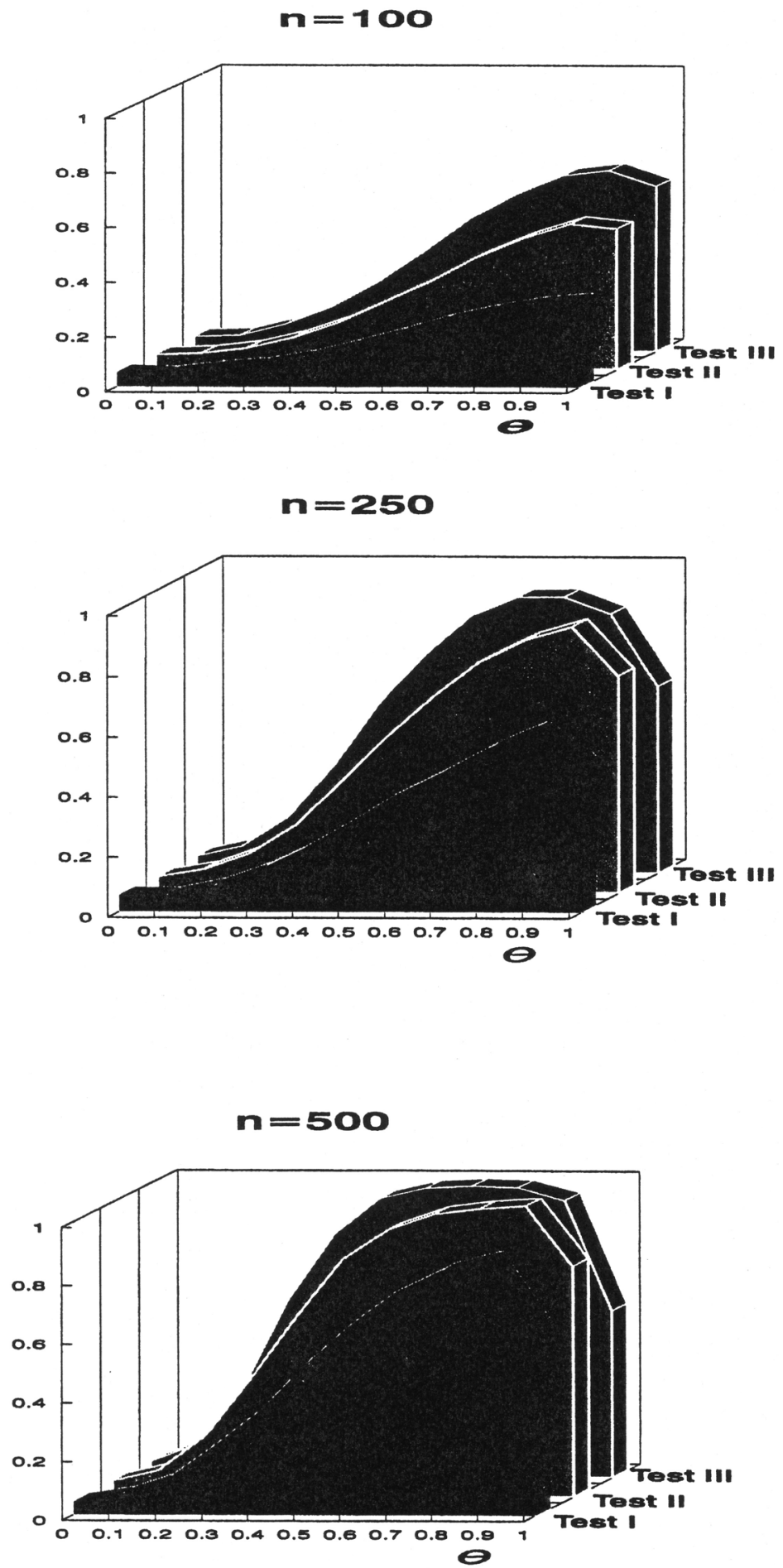

Fig. 2. Empirical frequency of rejection of null hypothesis. 
under the null hypothesis of non-predictability of returns, the corrected Student- $t$ ratio given by,

$$
t_{i}^{*}=\frac{\hat{\alpha}_{i}}{S_{\alpha_{i}}^{*}},
$$

has an approximate Student- $t$ distribution with $N-k$ degrees of freedom, $k$ being the number of estimated parameters in the regression.

In order to evaluate the efficiency of the corrected tests (denoted CTEST I, II and III, respectively), in comparison with the original uncorrected $t$-tests, Monte Carlo experiments, analogous to those previously described and using the same random numbers, have been performed. Figure 3 shows the efficiency gains (averaged for the sample sizes 100, 250 and 500), measured by the ratio of empirical frequencies of rejections of the null hypothesis, of the corrected tests relative to the corresponding uncorrected ones. The figure shows that, for low values of $\theta$, the gains in power of the corrected tests are meaningless, although clearly the highest for Test I. However, for $\theta$ approaching unity, substantial efficiency gains become evident. It seems that the proposed corrections are appropriate where there is a possibility of a lack of a meaningful quantity of spillovers between markets.

In practical applications, one difficulty is created by $v=\operatorname{Pr}(\omega>|\varepsilon| \mid \theta=0)$ not being directly observable, since it depends on the condition that $\theta=0$. Because the non-censored returns $\varepsilon_{t}$ are not fully observed, it is not possible to compute $v$ directly from the empirical data. The proposed procedure is based on approximating $v$ through the simulation of a relationship between the standard deviations of $\varepsilon_{t}$ and $r_{t}$ for the case where $\theta=0$. This involves performing a series of simulations

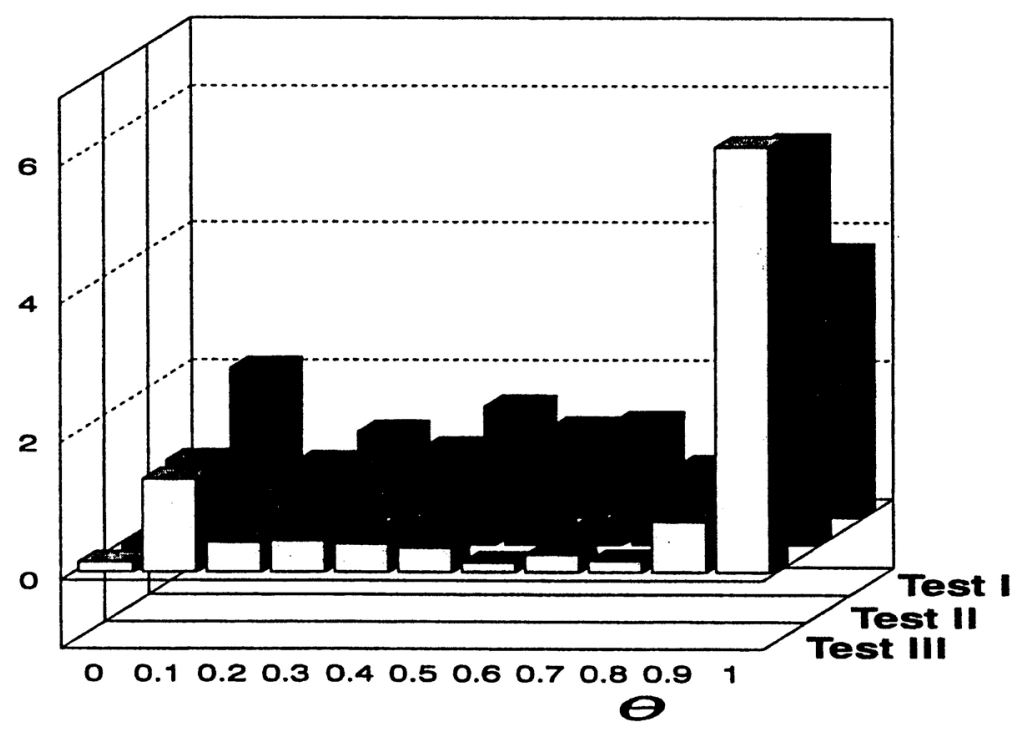

Fig. 3. Average efficiency gains of the corrected tests. 
on model (6)-(7) for $\omega=\ln (1.1), n=480$ and $\sigma_{\varepsilon}^{(j)}, j=1,2, \ldots, 50$, changing from 0.01 by 0.01 until 0.76 . For each $\sigma_{\varepsilon}^{(j)}, 100$ simulations of censored returns have been performed, with the computed average standard deviations denoted as $\hat{\sigma}_{r}^{(j)}$. Smoothing and interpolation make it possible to approximate $v$ as a function of the standard deviations of the truncated returns. Table 1 shows the estimated values of $v$ for some particular standard deviations. More detailed results and computation programs, written in GAUSS, are available on request.

The proposed way of computing the corrected test values is therefore as follows,

1. Compute standard deviations of observed returns.

2. Estimate $v$ for the appropriate standard deviation of observed returns from Table 1, approximating, if necessary.

3. Run the regressions (8), (9) and/or (10) and, using $v$, compute the corrected Student- $t$ statistics for CTEST I, II and/or III. Note that the mean of the observed returns has to be equal to zero; if it is not, adjust the returns for the mean.

Additionally, the estimated values of $v$ can be used for computation of a simple predictability test for returns. For $\theta=0$, that is where returns are not predictable, the expected value of frequency of barriers hits observed in the sample is equal to $v$. Hence, the straightforward $Z$ test for proportions is valid in large samples as a test for the hypothesis that $\theta=0$, i.e.:

$$
Z=\left(\frac{N^{h}}{N}-v\right) / \sqrt{\frac{v(1-v)}{N}} .
$$

The statistic $Z$ has an asymptotic standard normal distribution under the null hypothesis.

Table 1. Simulated and approximated two-sided probabilities of reaching the truncation point $(v) ; n=480$, censoring range $10 \%, \theta=0$

\begin{tabular}{llcc}
\hline$v$ & $\hat{\sigma}_{r}^{(j)}$ & $v$ & $\hat{\sigma}_{r}^{(j)}$ \\
\hline 0.0096 & 0.035 & 0.2571 & 0.065 \\
0.0208 & 0.040 & 0.3450 & 0.070 \\
0.0449 & 0.045 & 0.4524 & 0.075 \\
0.0789 & 0.050 & 0.5679 & 0.080 \\
0.1230 & 0.055 & 0.6935 & 0.085 \\
0.1843 & 0.060 & 0.8349 & 0.090 \\
\hline
\end{tabular}

$v=\operatorname{Pr}\left(w>\left|\varepsilon_{t}\right| \mid \theta=0\right)$ for Test II and Test III, and $v=\operatorname{Pr}\left(w>\varepsilon_{t} \mid \theta=0\right)=\operatorname{Pr}\left(-w<\varepsilon_{t} \mid\right.$ $\theta=0$ ) for Test I. $v$ is approximated through the simulation of a relationship between the standard deviations of $\varepsilon_{t}$ and $r_{t}$ from the system of equations (6), (7), for the case where $\theta=0$. $w=\ln (1.1), n=480$ and $\sigma_{\varepsilon}^{(j)}$ (standard deviation of $\varepsilon_{t}$ ) for $j=1,2, \ldots, 50$, is changed from 0.01 by 0.01 until 0.76 . For each $\sigma_{\varepsilon}^{(j)}, 100$ simulations of censored returns have been performed, where $\sigma_{\varepsilon}^{(j)}$ denotes the corresponding computed average standard deviation. 


\section{TESTING PREDICTABILITY FOR THE WARSAW STOCK EXCHANGE}

With the use of the above tests, in this section, we perform a preliminary analysis of ex-ante predictability of returns of shares for five companies, which are traded at the Warsaw Stock Exchange since it's establishment on 12th April 1991, and of one company for which shares were issued shortly after that date. The companies are: Exbud (EXB, construction services), Kable, (KAB, cable factory), Krosno, (KRO, glass factory), Próchnik, (PRO, clothes factory), Swarzedz, (SWA, furniture factory) and Tonsil (TON, electronics company). In this illustrative analysis, we investigate an early period of the Warsaw Stock Exchange, with data ranging over the period April 1991 to mid-May 1995. We focus on this period since the empirical frequency of barrier hits for the data following this period declines considerably, and therefore our assumption regarding the constancy of $v$ may not be maintained. The data used are for returns, on a session to session basis, with two sessions a week in 1991 and 1992, three sessions a week since the beginning of 1993, four sessions a week from mid-1994, and finally five sessions a week from the end of 1994. (Detailed descriptive and econometric analyses of the Warsaw Stock Exchange, including an analysis of possible calendar effects, can be found in Shields $(1997,1999)$, in addition to Gordon and Rittenberg (1995), Bolt and Milobedzki (1994a,b)). We do not use direct quantitative information concerning identification of the disequlibrium trading sessions. Instead, we have assumed that if returns were closer than $0.05 \%$ to its upper or lower boundary (that is, if the published price was equal or higher than 1.095 times the previous session price, or 0.905 or lower than the previous session price), the upper (lower) boundary was hit. This $0.05 \%$ tolerance limit allows for rounding errors of published prices. The source of the data was from detailed information published in Gazeta Bankowa (daily) and Rzeczypospolita (weekly). Data was collected and made available to us by the Macroeconomic and Financial Data Centre at the University of Gdansk. In a few instances missing observations were discarded. We also disregard the fact that in some cases transactions were suspended, (suspension of trade occurs if, for a given security, demand exceeds supply by more than fivefold or vice versa) and we censor to its upper or lower limit those returns which were allowed to go beyond that limit due to its occasional suspension.

Table 2 presents some descriptive statistics: the frequency of hits of the upper and lower barrier, the probability of a hit for $\theta=0$ (computed through interpolation from a more detailed version of Table 1), standard deviations of the observed series of logarithms of returns and that of non-censored returns for $\theta=0$ (that is, the simulated value of $\sigma_{\varepsilon}$ ) and the results of the computed Tests I and II in their uncorrected and corrected versions. For Test I, the parameters explaining the upper and lower hits (that is, $\beta_{1}$ and $\beta_{2}$ respectively) are tested separately. Also, the $Z$ test for $\theta=0$, based on the differences between the observed and theoretical frequencies of hits, has been computed.

The descriptive statistics show a relative homogeneity of the data set; the empirical frequencies of hits are of a similar order and standard deviations of the series are not far away from each other. The most volatile series seems to be those of returns from Tonsil and Kable, with the high frequencies of hits coupled with relatively high standard deviations. All the computed tests clearly indicate the predictability of the returns series and strongly suggest rejection of the null 
Table 2. Testing the predictability of return on Warsaw Stock Exchange, April 1991-May 1995

\begin{tabular}{|c|c|c|c|c|c|c|c|}
\hline & & TON & PRO & $\mathrm{KAB}$ & EXB & SWA & WOL \\
\hline $\begin{array}{l}\text { No. of } \\
\text { observations }\end{array}$ & & 471 & 476 & 476 & 476 & 467 & 464 \\
\hline $\begin{array}{l}\text { Frequency } \\
\text { of hits }\end{array}$ & & 0.25 & 0.24 & 0.29 & 0.22 & 0.26 & 0.20 \\
\hline$v$ & & 0.31 & 0.20 & 0.25 & 0.16 & 0.17 & 0.16 \\
\hline Std. Devs. & $\begin{array}{l}\text { Observed } \\
\text { Non-censored }\end{array}$ & $\begin{array}{l}0.068 \\
0.089\end{array}$ & $\begin{array}{l}0.061 \\
0.078\end{array}$ & $\begin{array}{l}0.064 \\
0.079\end{array}$ & $\begin{array}{l}0.058 \\
0.065\end{array}$ & $\begin{array}{l}0.059 \\
0.069\end{array}$ & $\begin{array}{l}0.058 \\
0.065\end{array}$ \\
\hline Test I & Uncorrected & -5.00 & -3.41 & -2.74 & -3.27 & -4.04 & -3.10 \\
\hline Lower & Corrected & -5.10 & -3.44 & -2.83 & -3.34 & -4.13 & -3.21 \\
\hline Test I & Uncorrected & 4.30 & 4.86 & 5.97 & 5.54 & 4.66 & 3.00 \\
\hline Upper & Corrected & 4.07 & 4.71 & 5.73 & 5.74 & 4.53 & 2.94 \\
\hline Test II & Uncorrected & 6.61 & 5.83 & 5.99 & 6.00 & 6.14 & 2.78 \\
\hline & Corrected & 6.37 & 5.85 & 5.93 & 6.24 & 6.08 & 2.90 \\
\hline$Z$ test & & 4.13 & 3.59 & 5.01 & 6.70 & 6.12 & 4.51 \\
\hline
\end{tabular}

Notes: See notes to Table 1 for the computation of $v$. The non-censored standard deviation refers to $\sigma_{\varepsilon}$ (i.e. for $\theta=0$ ) obtained from the simulation experiment on computing the corrected tests. The corrected versions of the tests uses the formula given in (12), the uncorrected uses equation (11), and both are computed for Test I and II in regressions (8) and (9), respectively. The Z-test is according to equation (13), which is asymptotically normally distributed under the null that $\theta=0$.

hypothesis that $\theta=0$. There is, generally, a correspondence between the high (low) frequency of hits and the corresponding values of $v$. This would suggest that the unknown values of $\theta$ might be approximately equal to each other for all the series investigated. In other words, the intertemporal spillover effects on these markets are of a similar magnitude.

\section{SUMMARY AND CONCLUSIONS}

Our results show that the proposed approach to modelling the predictability of returns for stock markets with disequilibrium trading might be of some practical importance. The method described in this paper can be used for the analysis of other stock exchanges on which similar trading systems are implemented, for instance, for the analysis of returns on the French Stock Exchange, on which price constraints are in operation. The results show that, contrary to the widespread opinion among the practitioners, the question of returns predictability on such markets is non-trivial and that the predictability should be tested rather than assumed. Our paper also shows that it is possible, although computer-intensive, to evaluate probabilities of reaching a disequilibrium state under the null hypothesis of non-predictability and then using these probabilities to correct the computed $t$-statistics in order to improve on the power of the tests. In future, research should concentrate on the analysis of ex-post rather than ex-ante predictability. Furthermore, the data generating process should be generalised in order to allow for the heavy-tailed distributions of the non-censored returns. This would make it feasible to analyse various speculative processes on stock exchanges with disequilibrium trading. 


\section{REFERENCES}

Baillie, R.T. (1989) Econometric tests of rationality and market efficiency, Econometric Reviews, 8, 151-86.

Benassy, J.P. (1982) The Economics of Market Disequilibrium. New York: Academic Press.

Bolt, T.W. and Milobedzki, P. (1994a) An empirical analysis of the Warsaw Stock Exchange, 1991-1993, Study Material No. 2, The Gdansk Institute for Market Economics. Gdansk.

Bolt, T.W. and Milobedzki, P. (1994b) The Warsaw Stock Exchange in the period 1991-1993: qualitative problems of its modelling, Economics of Planning, 27, 211-26.

Claessens, S., Dasgupta, S. and Glen, J. (1995) Return behavior in emerging stock markets, The World Bank Economic Review, 9, 153-74.

Drèze, J.H. (1975) Existence of the exchange equilibrium under price rigidities, International Economic Review, 16, 301-20.

Fama, E.F. (1965) The behaviour of stock market prices, Journal of Business, 38, 34-105.

Fama, E.F. (1970) Efficient capital markets: a review of theory and empirical work, Journal of Finance, 25, 383-417.

Fama, E.F. (1991) Efficient capital markets II, Journal of Finance, 46, 1575-617.

Gordon, B. and Rittenberg, L. (1995) The Warsaw Stock Exchange: a test of market efficiency, Comparative Economic Studies, 37, 1-27.

Granger, C.W.J. (1992) Forecasting stock market prices: lessons for forecasters, International Journal of Forecasting, 8, 3-13.

Grossman, S. and Stiglitz, J.E. (1980) On the impossibility of informationally efficient markets, American Economic Review, 70, 393-408.

LeRoy, S.F. (1989) Efficient capital markets and martingales, Journal of Economic Literature, 27, 1583-621.

Peters, E.E. (1991) Chaos and Order in the Capital Markets. New York: John Wiley.

Quandt, R.E. (1988) The Econometrics of Disequilibrium. Oxford: Basil Blackwell.

Richards, A.J. (1996) Volatility and predictability in national stock markets: how do emerging and mature markets differ?, International Monetary Fund Staff Papers, 43, 461-501.

Shields, K.K. (1997) Stock return volatility on emerging Eastern European markets, The Manchester School Supplement, 118-38.

Shields, K.K. (1999) An econometric analysis of emerging markets in Eastern Europe: the case of Poland. PhD Thesis, University of Leicester.

Williams, L.V. (1994) Random walks, fair games and efficient markets: the development of an idea. Occasional Paper in Economic No. 94/5, The Nottingham Trent University.

\section{Appendix: Predictability of returns on a market with disequilibrium trading}

In order to prove predictability it is sufficient to show that the expected value of returns conditional on the information on hitting the upper (lower) bound at a previous session is non-zero. Recalling the notation introduced in Section 2, the model (6)-(7), is given by:

$$
\begin{aligned}
& p_{t}^{*}=\theta p_{t-1}^{*}+(1-\theta) p_{t-1}+\epsilon_{t} ; \quad \theta \in[0,1] \\
& p_{t}=\left\{\begin{array}{cll}
p_{t}^{*} & \text { if } & \left|p_{t}^{*}-p_{t-1}\right|<\omega \\
\omega+p_{t-1} & \text { if } & p_{t}^{*}-p_{t-1}>\omega \\
-\omega+p_{t-1} & \text { if } & p_{t}^{*}-p_{t-1}<-\omega
\end{array}\right.
\end{aligned}
$$


Let us assume that in time $t-1$ an equilibrium trade took place, i.e. so that $p_{t-1}=p_{t-1}^{*}$, and in time $t$ the upper bound was hit, and therefore $\varepsilon_{t}>\omega$. Hence, the price generating process for time $t+1$ can be described as,

$$
\begin{aligned}
p_{t+1}^{*} & =\theta p_{t}^{*}+(1-\theta) p_{t}+\varepsilon_{t+1} \\
& =p_{t-1}^{*}+\theta \varepsilon_{t}+\omega-\theta \omega+\varepsilon_{t+1}, \\
p_{t+1} & =\left\{\begin{array}{ccc}
p_{t+1}^{*} & \text { if } & \left|p_{t+1}^{*}-p_{t}\right|<\omega \\
\omega+p_{t} & \text { if } & p_{t+1}^{*}-p_{t}>\omega \\
-\omega+p_{t} & \text { if } & p_{t+1}^{*}-p_{t}<-\omega
\end{array}\right.
\end{aligned}
$$

or,

$$
p_{t+1}=\left\{\begin{array}{ccl}
p_{t-1}^{*} & \text { if } & \left|\theta \varepsilon_{t}-\theta \omega+\varepsilon_{t+1}\right|<\omega \\
2 \omega+p_{t-1}^{*} & \text { if } & \theta \varepsilon_{t}-\theta \omega+\varepsilon_{t+1} \mid>\omega \\
p^{*} s_{t-1} & \text { if } & \theta \varepsilon_{t}-\theta \omega+\varepsilon_{t+1} \mid<-\omega
\end{array},\right.
$$

and the returns are given by:

$$
r_{t+1}=\left\{\begin{array}{cll}
\theta_{\varepsilon_{t}}-\theta \omega+\varepsilon_{t+1} & \text { if } & \left|\theta \varepsilon_{t}-\theta \omega+\varepsilon_{t+1}\right|<\omega \\
\omega & \text { if } & \theta \varepsilon_{t}-\theta \omega+\varepsilon_{t+1}>\omega . \\
-\omega & \text { if } & \theta \varepsilon_{t}-\theta \omega+\varepsilon_{t+1}<-\omega
\end{array} .\right.
$$

The sign of returns is positive if:

$$
0<\theta \varepsilon_{t}-\theta \omega+\varepsilon_{t+1}<\omega \text { or } \theta \varepsilon_{t}-\theta \omega+\varepsilon_{t+1}>\omega .
$$

The sign of returns is negative if:

$$
-\omega<\theta \varepsilon_{t}-\theta \omega+\varepsilon_{t+1}<0 \text { or } \theta \epsilon_{t}-\theta \omega+\varepsilon_{t}<-\omega .
$$

Thus the expected value of returns in time $t+1$, conditional on $\varepsilon_{t}>\omega$, can be written as:

$$
\begin{aligned}
E\left(r_{t+1} \mid \varepsilon_{t}>\omega\right)= & \omega \int_{\omega}^{\infty} f(x) \int_{\omega(\theta+1)-\theta x}^{\infty} f(y) d y d x+\left|\varepsilon_{t+1}\right| \int_{\omega}^{\infty} f(x) \int_{-\theta x+\theta \omega}^{\omega(\theta+1)-\theta x} f(y) d y d x \\
& +\left|\varepsilon_{t+1}\right| \int_{\omega}^{\infty} f(x) \int_{(\theta-1) \omega-\theta x}^{-\theta x+\theta \omega} f(y) d y d x-\omega \int_{\omega}^{\infty} f(x) \int_{-\infty}^{(\theta-1) \omega-\theta x} f(y) d y d x \\
= & \int_{\omega}^{\infty} f(x) \int_{\omega(\theta+1)-\theta x}^{(1-\theta) \omega+\theta x} f(y) d y d x+\left|\varepsilon_{t+1}\right| \int_{\omega}^{\infty} f(x) \\
& \times\left[\int_{-\theta x+\theta \omega}^{\omega(\theta+1)-\theta x} f(y) d y-\int_{(\theta-1) \omega-\theta x}^{-\theta x+\theta \omega} f(y) d y\right] d x
\end{aligned}
$$


which is not equal to zero if $0<\theta \leq 1$. For $\theta=0$, it simplifies to:

$$
\begin{aligned}
E\left(r_{t+1} \mid \varepsilon_{t}>\omega\right)= & \omega \int_{\omega}^{\infty} f(x) d x \int_{\omega}^{\infty} f(y) d y+\left|\varepsilon_{t+1}\right| \int_{\omega}^{\infty} f(x) d x \int_{\omega}^{\infty} f(y) d y d x \\
& -\left|\varepsilon_{t+1}\right| \int_{\omega}^{\infty} f(x) d x \int_{-\omega}^{\infty} f(y) d y-\omega \int_{\omega}^{\infty} f(x) \int_{-\omega}^{\infty} f(y) d y=0
\end{aligned}
$$

due to the symmetry of the constraint implying that the market is not predictable according to this framework. In this context, therefore, the expected value of returns conditional on information from the previous period relating to the constraint, is non-zero only if the value of the weight is non-zero. It is worth noting that the non-predictability of returns would not hold if the constraints become asymmetric and also if the process (6) is subject to a drift. 
Copyright of European Journal of Finance is the property of Routledge, Ltd. and its content may not be copied or emailed to multiple sites or posted to a listserv without the copyright holder's express written permission. However, users may print, download, or email articles for individual use. 